\title{
Level set methods for geometric inverse problems in linear elasticity
}

\author{
Hend Ben Ameur ${ }^{1,4}$, Martin Burger ${ }^{2,5}$ and Benjamin Hackl ${ }^{3}$ \\ ${ }^{1}$ SFB, ENIT, LAMSIN, BP 51, 1002 Tunis Belbedere, Tunisia \\ 2 Department of Mathematics, University of California, Los Angeles, 520 Portola Plaza, \\ Los Angeles, CA 90095-1555, USA \\ ${ }^{3}$ SBF F 013, Numerical and Symbolic Scientific Computing, Altenberger Str. 69, A-4040 Linz, \\ Austria \\ E-mail: hend.benameur@enit.rnu.tn,martin.burger@jku.at and hackl@sfb013.uni-linz.ac.at
}

Received 8 August 2003, in final form 15 January 2004

Published 15 March 2004

Online at stacks.iop.org/IP/20/673 (DOI: 10.1088/0266-5611/20/3/003)

\begin{abstract}
In this paper, we investigate the regularization and numerical solution of geometric inverse problems related to linear elasticity with minimal assumptions on the geometry of the solution. In particular, we consider the probably severely ill-posed reconstruction problem of a two-dimensional inclusion from a single boundary measurement. In order to avoid parametrizations, which would introduce a priori assumptions on the geometric structure of the solution, we employ the level set method for the numerical solution of the reconstruction problem. With this approach, we construct an evolution of shapes with a normal velocity chosen depending on the shape derivative of the corresponding least-squares functional in order to guarantee its descent. Moreover, we analyse penalization by perimeter as a regularization method, based on recent results on the convergence of Neumann problems and a generalization of Golab's theorem. The behaviour of the level set method and of the regularization procedure in the presence of noise are tested in several numerical examples. It turns out that reconstructions of good quality can be obtained only for simple shapes or for unreasonably low noise levels. However, it seems reasonable that the quality of reconstructions improves by using more than a single boundary measurement, which is an interesting topic for future research.
\end{abstract}

(Some figures in this article are in colour only in the electronic version)

\footnotetext{
4 This work was started during the visit to the JK university (Linz) as a research fellow 2002.
}

5 On leave from Johannes Kepler Universität Linz. 


\section{Introduction}

This paper is devoted to the regularization and numerical solution of some geometric inverse problems in linear elasticity. We study the inverse problem of identifying interfaces or inclusions (with possibly multiple connected components) from boundary measurements. We develop a rather general approach to such geometric inverse problems in linear elasticity, where the normal component of the stress tensor satisfies a homogeneous boundary condition on the unknown geometry (losing identifiablility, a non-homogeneous boundary condition is also possible). The boundary measurements in such applications are those of displacement on a part of the boundary where we have a boundary condition satisfied by the normal component of the stress tensor, too.

Geometric inverse problems, i.e. problems where the unknown is a geometric shape, have been investigated for around three decades (cf, e.g., $[15,28]$ ) with respect to theoretical and numerical aspects. A standard approach for the solution of such problems consists in parametrizing the shape and applying regularization methods directly to the parametrization (cf, e.g., [20-22]). This approach suffers from the limitation that considerable a priori knowledge on the structure and topology of the solution shape is required in order to obtain convergent approximations. In particular, any parametrization does not allow a change in the number of components, hence a shape can only be reconstructed if this number is known exactly. For the reasons described above, alternative approaches to the solution of shape reconstruction problems have been considered recently, such as the sampling methods (cf [27]) or the level set method (cf [45]). While the first method is based on properties of DirichletNeumann type operators and therefore requires a large number of measurements, the level set method can be applied also in the case of a single Dirichlet-Neumann measurement, which we therefore consider in this paper. The level set approach was introduced by Osher and Sethian [40] for evolving geometries, with the original aim of deriving fast algorithms for a flame propagation problem. The basic idea is to represent a shape implicitly as the zero level set of a continuous function and to use the correspondence of geometric variations of the shape and the solution of specific Hamilton-Jacobi equations for the level set function (cf section 4 for further details). Due to the implicit representation on an Eulerian grid, the level set approach does not introduce any a priori assumptions on the geometry and therefore is receiving growing attention in the context of geometric inverse problems (cf, e.g., [6, 7, 9, 10, $29,34,42,43,45]$ ) and shape optimization (cf, e.g., [3, 48]). In this paper, we shall investigate the application of level set methods in the context of elastic inclusion detection, which is a challenging problem both theoretically and numerically due to the Neumann condition on the boundary of the unknown shape.

By allowing rather general topology of the shape to be reconstructed, one also needs geometric regularization strategies independent of parametrizations. A frequently used approach is to add a multiple of the perimeter as a penalty to the least-squares functional. For problems that are only dependent on the shape via its indicator function, this approach is equivalent to total variation regularization (cf [44]) and can be analysed in the standard framework developed by Acar and Vogel [1]. For a problem with Neumann boundary condition on the unknown interface, the least-squares functional is not lower semicontinuous with respect to the standard topologies for the indicator function and hence, a completely different approach has to be used. It turns out that the problem is lower semicontinuous with respect to the Hausdorff distance of shapes if an appropriate weak formulation (in DenyLions spaces) of the Neumann problem is used. In this paper, we will prove that perimeter penalization is indeed a convergent regularization method in this case, by using methods of geometric measure theory and novel results on the generalization of Golab's theorem (i.e. 
the lower semicontinuity of the perimeter with respect to the Hausdorff-distance metric). Moreover, we investigate numerically the behaviour of this regularization strategy (imposing additional regularity assumptions for the geometry) in several examples and compare it to a direct regularization by a level-set evolution, where the regularizing effect comes from an early termination of the evolution.

Throughout this paper we consider a homogeneous isotropic linear elastic material, in a domain $\mathcal{D} \subset \mathbb{R}^{3}$, assuming that there exists a surface $\Gamma \subset \mathcal{D}$ that separates the domain into two disjoint open sets $\mathcal{D}_{1}$ and $\mathcal{D}_{2}$, i.e.

$$
\overline{\mathcal{D}}=\overline{\mathcal{D}}_{1} \cup \overline{\mathcal{D}}_{2}, \quad \Gamma=\partial \mathcal{D}_{1} \backslash \partial \mathcal{D}=\partial \mathcal{D}_{2} \backslash \partial \mathcal{D} .
$$

The linear elastic problem under consideration is specified by

$$
\begin{array}{ll}
\operatorname{div} \sigma(u)=0 & \text { in } \mathcal{D} \backslash \Gamma=\mathcal{D}_{1} \cup \mathcal{D}_{2} \\
\sigma(u) \cdot n=0 & \text { on } \Gamma,
\end{array}
$$

supplemented by appropriate boundary conditions (specified below) on $\partial \Omega, n$ denotes a normal vector to $\Gamma$. In the following, we will use the convention that this normal vector is in the outward normal direction of $\mathcal{D}_{1}$. The vector $u$ denotes the displacement and $\sigma(u)$ is the associated stress tensor, which is related via Hooke's law to the linearized strain tensor $\epsilon(u)$ via

$$
\sigma=\lambda \operatorname{tr} \epsilon I+2 \mu \epsilon .
$$

The linearized strain tensor $\epsilon(u)$ is given by

$$
\epsilon_{i j}(u)=\frac{1}{2}\left(\frac{\partial u_{i}}{\partial x_{j}}+\frac{\partial u_{j}}{\partial x_{i}}\right),
$$

tr denotes the trace of a matrix, and $\lambda, \mu$ are Lamé coefficients related to Young's modulus $E$ and the Poisson ratio $v$.

$$
\mu=\frac{E}{2(1+v)} \quad \lambda=\frac{E v}{(1-2 v)(1+v)} .
$$

The original identification process can be stated as

Inverse problem $3 D$. Identify the unknown shape $\Gamma$ by applying some prescribed load $g$ on $\Gamma_{N} \subset \partial \mathcal{D}$ and measuring the displacement induced by $g$ on some part $\Gamma_{M} \subset \Gamma_{N}$ with $\Gamma_{M}$ having a strictly positive measure.

We shall consider two two-dimensional cases derived from the three-dimensional problems for specific geometrical situations and special applied loads, the so-called planar and anti-planar cases. The full three-dimensional case shall be considered in future research.

Remark 1. When we require homogeneous Neumann conditions for the unknown boundary and higher regularity of the geometry (due to the use of shape derivatives), uniqueness and stability results can be shown under rather general geometric assumptions (cf [4]).

This paper is organized as follows: in the remaining part of the introduction we reduce the full three-dimensional problem (1.1) to two-dimensional problems, and formulate the inverse problems that will be investigated further in the following sections. The regularization of the identification problem with minimal assumptions on the regularity of the shape is discussed in section 2. In section 3, we derive a level set method to solve the regularized problems, respectively as a regularizing evolution itself. Moreover, we discuss the numerical solution of the elasticity problem by the immersed interface method. We test the numerical behaviour of the level set method and the regularization strategies in several numerical examples presented in section 4 , before we conclude and give an outlook for future work. 

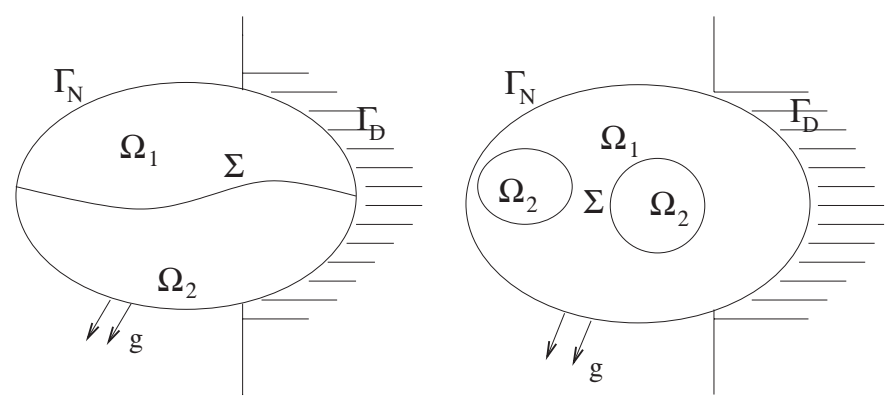

Figure 1. Different situations.

\subsection{Planar and anti-planar cases}

We assume that $\mathcal{D}=\Omega \times \mathbb{R}$, where $\Omega$ is a bounded domain in $\mathbb{R}^{2}, \Omega=\Omega_{1} \cup \Omega_{2}$ and $\Sigma=\partial \Omega_{1} \cap \partial \Omega_{2}$. The different possible geometric situations are shown in figure 1 .

Furthermore, we suppose that we apply a planar load $g$ and that the displacement $u: \Omega \times \mathbb{R} \longrightarrow \mathbb{R}^{3}$ depends only on $x_{1}$ and $x_{2}$. We split the initial 3D problem (1.1) into two problems: a planar strain one, where we consider $u\left(x_{1}, x_{2}\right)=\left(u_{1}\left(x_{1}, x_{2}\right), u_{2}\left(x_{1}, x_{2}\right), 0\right)$, and an anti-planar problem depending only on the third component $u\left(x_{1}, x_{2}\right)=\left(0,0, u_{3}\left(x_{1}, x_{2}\right)\right)$.

In the planar strain case, we obtain a stress tensor $\sigma$ satisfying $\sigma_{3, \ell}=\sigma_{\ell, 3}=0, \ell=1,2$. The choice of $g$ with a third component equal to zero will allow us to obtain a twodimensional problem similar to the one corresponding to the plane stress case where $\sigma_{3, \ell}=\sigma_{l, \ell}=0, l=1,2,3$. We refer to both as 'planar case' and rewrite (1.1) for the planar case as

$$
\begin{array}{ll}
\operatorname{div} \sigma(u)=0 & \text { in } \Omega \backslash \Sigma \\
\sigma(u) \cdot n=g & \text { on } \Gamma_{N} \\
\sigma(u) \cdot n=0 & \text { on } \Sigma \\
u=0 & \text { on } \Gamma_{D},
\end{array}
$$

where $\left\{\Gamma_{D}, \Gamma_{N}\right\}$ is a partition of the boundary of $\Omega$ supporting Dirichlet and Neumann boundary conditions. The identification problem can then be stated as

Inverse problem, planar case. Identify the unknown interface $\Sigma$ from a measurement of the displacement $u$ on $\Gamma_{M} \subset \Gamma_{N}$ (with $\Gamma_{M}$ having a strictly positive measure), where $u=\left(u_{1}, u_{2}\right)$ is the solution of (1.3) with the constitutive law (1.2).

In the anti-planar case, the original linear elasticity problem (1.1) reduces to a boundary value problem for a single Laplace equation:

$$
\begin{array}{ll}
\mu \Delta u_{3}=0 & \text { in } \Omega \backslash \Sigma \\
\mu \frac{\partial u_{3}}{\partial n}=g & \text { on } \Gamma_{N} \\
\mu \frac{\partial u_{3}}{\partial n}=0 & \text { on } \Sigma \\
u_{3}=0 & \text { on } \Gamma_{D},
\end{array}
$$

where $\mu$ is a Lamé constant.

For simplicity, we denote $u_{3}$ by $u$ in (1.4) when the anti-planar case is concerned. The associated identification problem can then be formulated as 
Inverse problem, anti-planar case. Identify the unknown interface $\Sigma$ from a measurement of the vertical displacement $u$ on $\Gamma_{M} \subset \Gamma_{N}$ (with $\Gamma_{M}$ having a strictly positive measure), where $u$ is the solution of (1.4).

\subsection{Weak formulations of the direct problem}

In this section, we introduce weak formulations of the direct problems (1.3) and (1.4), under minimal assumptions on the regularity of the interface $\Sigma$. In order to allow for general Hausdorff-measurable interfaces $\Sigma$, we use a framework in Deny-Lions spaces, recently used for the mathematical modelling and analysis of crack growth by Chambolle [11], Dal Maso and Toader [35] and Giacomini [19]. For smooth interfaces $\Sigma$, this solution concept coincides with the standard framework for weak solutions in Sobolev spaces.

In the following, we assume that $\Sigma$ is a compact subset of $\Omega$ with a finite number of connected components, which are measurable with respect to the Hausdorff measure $\mathcal{H}^{1}(\Sigma)$ (we refer to [18] and [36] for details on the definition and properties of Hausdorff measures). Under such general condition, a standard weak formulation is no longer suitable, since one cannot define Sobolev spaces such as $H^{1}(\Omega \backslash \Sigma)$ in a classical way. Therefore, we introduce a generalization of Sobolev spaces for non-smooth domains as follows: If $A$ is an open subset of $\mathbb{R}^{2}$ (keeping in mind $A=\Omega \backslash \Sigma$ ), the Deny-Lions space $L^{1,2}(A)$ is defined by

$$
L^{1,2}(A):=\left\{u \in W_{\text {loc }}^{1,2}(A) \mid \nabla u \in L^{2}\left(A ; \mathbb{R}^{2}\right)\right\} .
$$

Note that for a regular $A$, the Deny-Lions space coincides with the usual Sobolev space $H^{1}(A)$, but it is a strict superset for non-regular sets. However, since we assume that $\Sigma$ is a compact subset of $\Omega$ and $\partial \Omega$ is regular, each element in $L^{1,2}(\Omega \backslash \Sigma)$ is embedded in a local $H^{1}$-space in a neighbourhood of the boundary so that we can define traces on $\partial \Omega$ in a standard way. Finally, we define the Deny-Lions space $L^{1,2}\left(A ; \mathbb{R}^{2}\right)$ (cf, e.g., [19] for further details).

$$
L^{1,2}\left(A ; \mathbb{R}^{2}\right):=\left\{u \in W_{\text {loc }}^{1,2}\left(A ; \mathbb{R}^{2}\right) \mid \nabla u \in L^{2}\left(A ; \mathbb{R}^{2 \times 2}\right)\right\} .
$$

In the planar case, we specify the space of admissible vertical displacements as

$$
\mathcal{U}:=\left\{u \in L^{1,2}(\Omega \backslash \Sigma)|u|_{\Gamma_{D}}=0\right\} .
$$

The weak formulation of the anti-planar case consists in finding $u \in \mathcal{U}$ such that

$$
a(u, v):=\int_{\Omega \backslash \Sigma} \nabla u \cdot \nabla v \mathrm{~d} x=\int_{\Gamma_{N}} g v \mathrm{~d} s=:\langle G, v\rangle \quad \forall v \in \mathcal{U} .
$$

The solution of this problem is also the unique minimizer of the variational problem

$$
a(u, u)-2\langle G, u\rangle \rightarrow \min _{u \in \mathcal{U}}
$$

In the planar case, we can rewrite the variational problem in an analogous way, now with the vectorial Deny-Lions space

$$
\mathcal{U}:=\left\{u \in L^{1,2}\left(\Omega \backslash \Sigma ; \mathbb{R}^{2}\right)|u|_{\Gamma_{D}}=0\right\},
$$

and the variational form

$$
a(u, v):=\int_{\Omega \backslash \Sigma} \sigma(u): \epsilon(v) \mathrm{d} x=\int_{\Gamma_{N}} g \cdot v \mathrm{~d} s=:\langle G, v\rangle \quad \forall v \in \mathcal{U},
$$

with $\sigma$ and $\epsilon$ defined as above, and $\sigma: \epsilon=\sum_{i, j} \sigma_{i j} \epsilon_{i j}$.

Finally, we remark that for these weak formulations, existence and uniqueness of solutions for given interface $\Sigma$ is guaranteed by the results in [19]. In particular, the shape-to-output map $\left.\Sigma \mapsto u\right|_{\Gamma_{M}}$ is well defined, a fact that we shall use subsequently without further notice. 


\section{Geometric regularization}

Usually inclusion and interface identification problems are ill-posed. Even when, for our problem class, a local stability result was proved in [4] that might indicate the well posedness of the inverse problems under consideration, these stability results, however, are valid only under strong additional assumptions on the shape, and only yield local and directional information. In particular, they provide no information about the stability with respect to general perturbations of the output data in $L^{2}\left(\Gamma_{M}\right)$, in whose norm we measure the error. The somehow minimal requirements for well posedness in this output space would mean the existence of a solution to the least-squares problem

$$
J_{0}(\Sigma)=\left\|u-\theta^{\delta}\right\|_{L^{2}\left(\Gamma_{M}\right)}^{2} \rightarrow \min _{\Sigma \in \mathcal{K}}
$$

in some appropriate class of shapes $\mathcal{K}$ together with weak stability of the minimizer with respect to perturbations of the data $\theta^{\delta}$. As observed from the following example, weak stability in a general class of shapes does not hold:

Example 2.1. Let $\Omega=[0,1] \times[-1,1], \Gamma_{M}=[0,1] \times\{1\}$ and define a sequence of interfaces $\Sigma_{n}$ via

$$
\Sigma_{n}=\left\{\left(x_{1}, x_{2}\right) \mid x_{2}=\frac{1}{2} \sin n \pi x_{1}\right\},
$$

with associated states $u_{n}$ solving (1.4) and $f_{n}=\left.u_{n}\right|_{\Gamma_{M}}$. Moreover, we pose homogeneous Dirichlet conditions on $\Gamma_{D}=\{0,1\} \times(-1,1)$ and some Neumann boundary conditions $g$ on $\Gamma_{N}=[0,1] \times\{-1,1\}$, non-vanishing on $\Gamma_{M}$ only. If $\psi$ is the unique solution of $\Delta \psi=0$ in $\Omega$ subject to these boundary conditions, then by the definition of $u_{n}$ we obtain

$$
\int_{\Omega}\left|\nabla u_{n}\right|^{2} \mathrm{~d} x+2 \int_{\Gamma_{N}} g u_{n} \mathrm{~d} s \leqslant \int_{\Omega}|\nabla \psi|^{2} \mathrm{~d} x+2 \int_{\Gamma_{N}} g \psi \mathrm{d} s:=C .
$$

In particular, we obtain for the integral over $S=(0,1) \times\left(\frac{1}{2}, 1\right)$

$$
\int_{S}\left|\nabla u_{n}\right|^{2} \mathrm{~d} x+2 \int_{\Gamma_{N}} g u_{n} \mathrm{~d} s \leqslant C .
$$

For functions in $H^{1}(S)$ with homogeneous Dirichlet values on $\{0,1\} \times\left(\frac{1}{2}, 1\right)$ the Poincaré inequality shows that the first integral is an equivalent norm to the $H^{1}$-norm and if $\|g\|_{H^{-\frac{1}{2}}\left(\Gamma_{M}\right)}$ is sufficiently small, we obtain

$$
\int_{S}\left|\nabla u_{n}\right|^{2} \mathrm{~d} x \leqslant \frac{C}{2}
$$

Thus, the restriction of $u_{n}$ is uniformly bounded in $H^{1}(S)$ and due to the compactness of the trace operator from $H^{1}(S)$ to $L^{2}\left(\Gamma_{M}\right)$, there exists a subsequence of $f_{n}$ converging to some $f$ in $L^{2}\left(\Gamma_{M}\right)$, but the sequence $\Sigma_{n}$ has no subsequence converging to a curve with finite Hausdorff measure $\mathcal{H}^{1}$. This example shows that without severe restrictions, the problem is ill-posed even in any class of $\Sigma$ piecewise in $C^{k}$ (for any $k \in \mathbb{N}$ ) and $\mathcal{H}^{1}$-measurable, since the $\Sigma_{n}$ constructed above are even analytic.

As usual for ill-posed problems, we have to introduce a regularization approach in order to compute stable approximations of the solution in the presence of data noise (cf [16] for an overview on this subject), In the following parts, we consider two different approaches to the regularization of (1.1) and (1.2). The first one is a penalization approach motivated by the Mumford-Shah functional used frequently in image processing (cf [36]), while the second consists in the early stopping of an evolution in artificial time, motivated by asymptotic regularization of inverse problems. 


\subsection{Penalization by perimeter}

A common approach to the stabilization of inverse interface problems is to add the perimeter (i.e. the Hausdorff measure $\mathcal{H}^{1}$ ) of $\Sigma$ as a penalty to the output functional, i.e. to minimize

$$
J_{\alpha}(\Sigma)=\frac{1}{2} \int_{\Gamma_{M}}\left|u-\theta^{\delta}\right|^{2} \mathrm{~d} s+\alpha \mathcal{H}^{1}(\Sigma) \rightarrow \min _{\Sigma \in \mathcal{K}}
$$

where $\mathcal{K}$ denotes the class of compact subsets of $\Omega$. Penalization by perimeter is well analysed in the context of the Mumford-Shah functional in image processing, where $\Sigma$ represents the discontinuity set of the function $u$, whose $L^{2}$-distance to some given function is to be measured (see [36] for a comprehensive overview). Recently, this strategy has been used in the context of shape optimization or geometric inverse problems (cf [24]). Problems with the analysis of this regularization strategy are caused, in particular, by the fact that the perimeter itself is not lower semicontinuous in the class $\mathcal{K}$ with respect to the Hausdorff metric, which seems to be natural if the convergence of shapes is considered.

The lower semicontinuity on the subclass $\hat{\mathcal{K}}_{1}$ of simply connected, $\mathcal{H}^{1}$-measurable compact sets is provided by a classical result in geometric measure theory, called Golab's theorem (cf [36]). From recent results by Dal Maso and Toader [35] and in particular by Giacomini [19], the lower semicontinuity of the perimeter is guaranteed on the more interesting class $\hat{\mathcal{K}}_{m}$ of $\mathcal{H}^{1}$-measurable compact sets with at most $m$ connected components, which we shall use in our analysis below:

Theorem 2.2 (35, corollary 3.3). Let $\left(\Sigma_{n}\right)$ be a sequence in $\hat{\mathcal{K}}_{m}$ converging to $\Sigma$ in the Hausdorff metric. Then $\Sigma \in \hat{\mathcal{K}}_{m}$ and

$$
\mathcal{H}^{1}(\Sigma \cap \Omega) \leqslant \liminf _{n \rightarrow \infty} \mathcal{H}^{1}\left(\Sigma_{n} \cap \Omega\right) .
$$

Moreover, the results in these papers allow us to also deduce the lower semicontinuity of the output functional and thus the analysis of this regularization strategy in a similar way to the analysis of standard Tikhonov regularization for nonlinear inverse problems (cf [46, 17]), respectively to the analysis of the so-called total variation $(T V)$ regularization (cf $[44,1]$ ). The proof of the lower semicontinuity of the output functional is the only property in this section for which we have to consider the two different cases of planar and anti-planar strain. We start with the anti-planar one, for which we use the results of Dal Maso and Toader [35]:

Proposition 2.3 (lower semicontinuity in the anti-planar case). The functional

$$
J_{0}: \Sigma \mapsto \int_{\Gamma_{M}}|u-\theta|^{2} \mathrm{~d} s,
$$

is lower semicontinuous on $\hat{\mathcal{K}}_{m}$ for arbitrary $\theta \in L^{2}\left(\Gamma_{M}\right)$, i.e. for any sequence $\left(\Sigma_{n}\right)$ in $\hat{\mathcal{K}}_{m}$ converging to $\hat{\Sigma}$ in the Hausdorff metric, the solutions $u_{n}$, respectively $\hat{u}$, of (1.5), (1.6) with $\Sigma$ replaced by $\Sigma_{n}$, respectively $\hat{\Sigma}$, satisfy

$$
\int_{\Gamma_{M}}|\hat{u}-\theta|^{2} \mathrm{~d} s \leqslant \liminf _{n \rightarrow \infty} \int_{\Gamma_{M}}\left|u_{n}-\theta\right|^{2} \mathrm{~d} s .
$$

Proof. From Dal Maso and Toader [35 theorem 5.1], the gradients of the solutions $u_{n}$ and $\hat{u}$ (extended by zero on $\Sigma_{n}$ and $\hat{\Sigma}$, respectively) satisfy $\nabla u_{n} \rightarrow \nabla \hat{u}$ strongly in $L^{2}\left(\Omega ; \mathbb{R}^{2}\right)$. Thus, for some open set $U$ with $\Gamma_{M} \subset \partial U$, we obtain that $u_{n} \rightarrow u$ in $H^{1}(U)$ and thus, a standard trace theorem implies $\left.\left.u_{n}\right|_{\Gamma_{M}} \rightarrow \hat{u}\right|_{\Gamma_{M}}$ in $H^{\frac{1}{2}}\left(\Gamma_{M}\right) \hookrightarrow L^{2}\left(\Gamma_{M}\right)$. Together with the lower semicontinuity of the $L^{2}$-norm this implies the assertion. 
The proof in the planar case can be carried out in a completely analogous way, now using the results of Giacomini [19, in particular, lemma 6.1] and will thus be omitted here:

Proposition 2.4 (lower semicontinuity in the planar case). The functional

$$
J_{0}: \Sigma \mapsto \int_{\Gamma_{M}}|u-\theta|^{2} \mathrm{~d} s
$$

is lower semicontinuous on $\hat{\mathcal{K}}_{m}$ for arbitrary $\theta \in L^{2}\left(\Gamma_{M} ; \mathbb{R}^{2}\right)$, i.e. for any sequence $\left(\Sigma_{n}\right)$ in $\hat{\mathcal{K}}_{m}$ converging to $\hat{\Sigma}$ in the Hausdorff metric, the solutions $u_{n}$ respectively $\hat{u}$ of $(1.2),(1.7)$ with $\Sigma$ replaced by $\Sigma_{n}$ respectively $\hat{\Sigma}$, satisfy

$$
\int_{\Gamma_{M}}|\hat{u}-\theta|^{2} \mathrm{~d} s \leqslant \liminf _{n \rightarrow \infty} \int_{\Gamma_{M}}\left|u_{n}-\theta\right|^{2} \mathrm{~d} s .
$$

In the following, we will not distinguish the planar and the anti-planar cases. By the data $f \in L^{2}\left(\Gamma_{M}\right)$ we denote either scalar- or vector-valued functions. Moreover, for each $\Sigma$ with some index, the function $u$, with the same index, will either denote the solution of (1.5), (1.6) or (1.2), (1.7), which we will use without further notice.

The first two results are concerned with the existence and (set-valued) stability of minimizers of (2.1) for $\alpha>0$ :

Proposition 2.5 (existence of a minimizer). For any $\theta^{\delta} \in L^{2}\left(\Gamma_{M}\right)$ and any $\alpha>0$ there exists a minimizer $\Sigma_{\alpha}^{\delta} \in \hat{\mathcal{K}}_{m}$ of $(2.1)$.

Proof. Let $\left(\Sigma_{n}\right)$ be a minimizing sequence in $\hat{\mathcal{K}}_{m}$ (which exists since the functional is bounded below by zero), then due to the boundedness of $\Omega$, there exists a convergent subsequence in the Hausdorff metric $d_{H}$ with limit $\Sigma_{\alpha}^{\delta} \in \hat{\mathcal{K}}_{m}$. From the lower semicontinuity results in the preceding propositions, we deduce that

$$
\frac{1}{2} \int_{\Gamma_{M}}\left|u_{\alpha}^{\delta}-\theta^{\delta}\right|^{2} \mathrm{~d} s+\alpha \mathcal{H}^{1}\left(\Sigma_{\alpha}^{\delta}\right) \leqslant \frac{1}{2} \int_{\Gamma_{M}}\left|u_{n}-\theta^{\delta}\right|^{2} \mathrm{~d} s+\alpha \mathcal{H}^{1}\left(\Sigma_{n}\right),
$$

and thus, $\Sigma_{\alpha}^{\delta}$ is a solution of (2.1).

Proposition 2.6 (stability). Let $\alpha>0$, let $\left(\theta_{n}\right)$ be a sequence in $L^{2}\left(\Gamma_{M}\right)$ converging to $\theta^{\delta}$ and let $\left(\Sigma_{n}\right)$ be the associated sequence of minimizers in $\hat{\mathcal{K}}_{m}$ of $(2.1)$ with data $\theta_{n}$. Then, there exists a subsequence of $\left(\Sigma_{n}\right)$ convergent in the Hausdorff metric $d_{H}$ and the limit of each convergent subsequence is a solution of (2.1) with data $\theta^{\delta}$.

Proof. First of all, the existence of the minimizers $\Sigma_{n} \in \hat{\mathcal{K}}_{m}$ is guaranteed by proposition 2.5. The existence of a convergent subsequence in the Hausdorff metric $d_{H}$ follows from $\Sigma_{n} \subset \Omega$ and the boundedness of $\Omega$. Now let $\Sigma_{n}$ denote a converging subsequence with limit $\Sigma_{\alpha}^{\delta}$ and let $u_{n}, u_{\alpha}^{\delta}$ denote the associated sequence of states. Then, from the strong convergence $\theta_{n} \rightarrow \theta^{\delta}$ and the definition of $u_{n}$ and $\Sigma_{n}$ we deduce the inequality

$$
\begin{aligned}
\frac{1}{2} \int_{\Gamma_{M}}\left|u_{\alpha}^{\delta}-\theta^{\delta}\right|^{2} \mathrm{~d} s+\alpha \mathcal{H}^{1}\left(\Sigma_{\alpha}^{\delta}\right) & \leqslant \liminf _{n \rightarrow \infty} \frac{1}{2} \int_{\Gamma_{M}}\left|u_{n}-\theta^{\delta}\right|^{2} \mathrm{~d} s+\alpha \mathcal{H}^{1}\left(\Sigma_{n}\right) \\
& \leqslant \liminf _{n \rightarrow \infty} \frac{1}{2} \int_{\Gamma_{M}}\left|u_{n}-\theta_{n}\right|^{2} \mathrm{~d} s+\alpha \mathcal{H}^{1}\left(\Sigma_{n}\right) \\
& \leqslant \lim _{n \rightarrow \infty} \frac{1}{2} \int_{\Gamma_{M}}\left|u-\theta_{n}\right|^{2} \mathrm{~d} s+\alpha \mathcal{H}^{1}(\Sigma) \\
& \leqslant \frac{1}{2} \int_{\Gamma_{M}}\left|u-\theta^{\delta}\right|^{2} \mathrm{~d} s+\alpha \mathcal{H}^{1}(\Sigma)
\end{aligned}
$$


for any $\Sigma \in \hat{\mathcal{K}}_{m}$ with associated state $u$. Hence, the limit $\Sigma_{\alpha}^{\delta}$ is also a solution of problem (2.1).

Now, we are able to prove the main result of this section, namely that penalization by perimeter is a convergent regularization method in $\hat{\mathcal{K}}_{m}$ equipped with the Hausdorff metric $d_{H}$ :

Theorem 2.7 (convergence). Suppose that there exists $\Sigma^{*} \in \hat{\mathcal{K}}_{m}$ such that the according solution $u^{*}$ of (1.5), (1.6) or (1.2), (1.7), respectively, satisfies $\left.u^{*}\right|_{\Gamma_{M}}=\theta$. Let $\theta^{\delta}$ be such that

$$
\left\|u^{*}-\theta^{\delta}\right\|_{L_{2}\left(\Gamma_{M}\right)} \leqslant \delta
$$

holds and let $\alpha$ be chosen such that

$$
\alpha \rightarrow 0, \quad \frac{\delta^{2}}{\alpha} \rightarrow 0 \quad \text { as } \delta \rightarrow 0 .
$$

Then, for the according solutions $\Sigma_{\alpha}^{\delta}$ of (2.1), there exists a subsequence convergent in the Hausdorff metric $d_{H}$ and the limit $\hat{\Sigma}$ of every convergent sequence is a minimum-perimeter solution of the identification problem, i.e. the associated displacement $\hat{u}$ satisfies $\left.\hat{u}\right|_{\Gamma_{M}}=\theta$ and for all other solutions $\Sigma$, we have that

$$
\mathcal{H}^{1}(\hat{\Sigma}) \leqslant \mathcal{H}^{1}(\Sigma)
$$

Proof. Again by boundedness, there exists a subsequence $\left(\Sigma_{n}\right):=\left(\Sigma_{\alpha_{n}}^{\delta_{n}}\right)$ converging in the Hausdorff metric $d_{H}$. Now let $\left(\Sigma_{n}\right)$ be such a convergent subsequence with limit $\hat{\Sigma}$. By the definition of $\Sigma_{n}$ we obtain by comparison with $\Sigma^{*}$ that

$$
\frac{1}{2} \int_{\Gamma_{M}}\left|u_{n}-\theta^{\delta_{n}}\right|^{2} \mathrm{~d} s+\alpha_{n} \mathcal{H}^{1}\left(\Sigma_{n}\right) \leqslant \frac{1}{2} \int_{\Gamma_{M}}\left|\theta-\theta^{\delta_{n}}\right|^{2} \mathrm{~d} s+\alpha_{n} \mathcal{H}^{1}\left(\Sigma^{*}\right)=\frac{\delta_{n}^{2}}{2}+\alpha_{n} \mathcal{H}^{1}\left(\Sigma^{*}\right) .
$$

Division by $\alpha_{n}$ and the assumption $\frac{\delta_{n}^{2}}{\alpha_{n}} \rightarrow 0$ shows that $\mathcal{H}^{1}\left(\Sigma_{n}\right)$ is uniformly bounded and the lower semicontinuity of the Hausdorff measure yields $\mathcal{H}^{1}(\hat{\Sigma}) \leqslant \mathcal{H}^{1}\left(\Sigma^{*}\right)$. Moreover, as $\delta_{n} \rightarrow 0$, we obtain

$$
\frac{1}{2} \int_{\Gamma_{M}}|\hat{u}-\theta|^{2} \mathrm{~d} s \leqslant \liminf _{n \rightarrow \infty} \frac{1}{2} \int_{\Gamma_{M}}\left|u_{n}-\theta^{\delta_{n}}\right|^{2} \mathrm{~d} s \leqslant \liminf _{n \rightarrow \infty} \frac{\delta_{n}^{2}}{2}+\alpha_{n} \mathcal{H}^{1}\left(\Sigma^{*}\right)=0 .
$$

Thus, $\hat{\Sigma}$ is a solution of the identification problem and since the solution $\Sigma^{*}$ is arbitrary, it is also a minimum-perimeter solution.

We finally remark that we obtain strong convergence by a standard principle, if the minimum-perimeter solution is unique. In particular this would be guaranteed by an identifiability result in the class $\hat{\mathcal{K}}_{m}$, but so far we can only give such a result for disjoint unions of smooth curves (cf [4]).

\section{Numerical solution}

In this section, we discuss a level set approach to the solution of the identification problem, respectively to the optimization problem (2.1) obtained from penalization by perimeter. Furthermore, we discuss the solution of the arising Hamilton-Jacobi equation as well as the solution of the elliptic problem with moving interface. 


\subsection{Level set methods}

The level set approach to geometric motion has been introduced by Osher and Sethian [40]. Its main idea is to represent an evolving front as the zero level set of a continuous function, i.e.

$$
\Sigma(t)=\{x \in \Omega \mid \phi(x, t)=0\} .
$$

A weak formulation of geometric motion with normal speed $V_{n}$ is given by the HamiltonJacobi equation

$$
\frac{\partial \phi}{\partial t}+V_{n}|\nabla \phi|=0 \quad \text { in } \mathbb{R}^{d} \times \mathbb{R}^{+}
$$

in the sense that a viscosity solution for this Hamilton-Jacobi equation (3.1) has to be computed. We refer to the monograph by Lions [33] and the paper by Crandall et al [13] for details on the notion of viscosity solution. The function $V_{n}$ in the Hamilton-Jacobi equation (3.1) is an extension of the velocity from the front to the whole $\mathbb{R}^{N}$ (or subset of $\mathbb{R}^{N}$ being the computational domain and including the interface).

The basic idea of level set methods for inverse and optimization problems (cf [6, 24, 39, $42,43,45])$ is to choose the velocity in such a way that a decrease of the objective functional is achieved, which resembles the classical speed method in shape optimization (cf [37, 49]), but the weak formulation via the level set method allows for more general evolution and in particular for topological changes such as splitting or merging of domains. We restrict our attention again to the anti-planar case, noting that analogous reasoning is possible for the planar case.

In the following, we discuss the construction of level set methods based on the original sharp interface model for the state equation. The basic tool for the choice of the velocity is the shape derivative in the direction of a normal velocity $V_{n}$ (cf $[14,49]$ ), which coincides with the time derivative of the objective functional $J_{\alpha}$, when the shape is evolved according to the geometric motion defined by (3.1). To get a well-defined shape derivative, we need some additional regularity constraints for the level set $\{\phi(t)=0\}$, namely that $\{\phi(t)=0\} \in C^{2}$. In general, we cannot guarantee that these regularity constraints for the level set are fulfilled during the level set evolution which is a general bottleneck of level set methods using shape derivatives and provides a rich source for future research.

For the anti-planar case (1.4) as well as for the planar case (1.3), the shape derivatives for the above regular sets are well known (cf (49, proposition 3.3 and theorem 3.11)). We denote only the anti-planar case:

Theorem 3.1. Let $\Sigma \in C^{2}$, $u$ be the solution to the anti-planar case (1.4), $V_{n} \in C^{1}(\Sigma)$ then the shape derivative $u^{\prime}$ is determined by the unique solution of

$$
\begin{array}{ll}
\Delta u^{\prime}=0 & \text { in } \Omega \backslash \Sigma \\
\frac{\partial u^{\prime}}{\partial n}=0 & \text { on } \Gamma_{N} \\
\frac{\partial u^{\prime}}{\partial n}=-\operatorname{div}_{T}\left(V_{n} \nabla_{T} u\right) & \text { on } \Sigma \\
u^{\prime}=\frac{\partial u}{\partial n} V_{n} & \text { on } \Gamma_{D},
\end{array}
$$

where $\operatorname{div}_{T}$ and $\nabla_{T}$ are defined via: let $U$ be an open neigbourhood of $\Sigma$ and $Y \in C^{1}\left(U, \mathbb{R}^{n}\right)$, then

$$
\operatorname{div}_{T} Y=\left.\operatorname{div} Y\right|_{\Sigma}-\left.\langle D Y \cdot n, n\rangle\right|_{\Sigma} \quad \nabla_{T} Y=\left.\nabla Y\right|_{\Sigma}-\left.\frac{\partial Y}{\partial n}\right|_{\Sigma} n
$$


Using this, the time derivative of $J(\Sigma(t))$ under a geometric motion with normal velocity is given by

$$
\frac{\mathrm{d}}{\mathrm{d} t} J_{\alpha}(\Sigma(t))=\int_{\Gamma_{M}}\left(u-\theta^{\delta}\right) u^{\prime} \mathrm{d} s+\alpha \int_{\Sigma} V_{n} \kappa \mathrm{d} \mathcal{H}^{1},
$$

where $\kappa$ denotes the mean curvature of $\Sigma$, and $u^{\prime}$ is the solution of (3.2). Introducing the adjoint problem

$$
\begin{aligned}
& \Delta w=0 \quad \text { in } \Omega \backslash \Sigma \\
& \frac{\partial w}{\partial n}=\chi_{\Gamma_{M}}\left(u-\theta^{\delta}\right) \quad \text { on } \Gamma_{N} \\
& \frac{\partial w}{\partial n}=0 \quad \text { on } \Sigma \\
& w=0 \quad \text { on } \Gamma_{D} \text {, }
\end{aligned}
$$

where $\chi_{\Gamma_{M}}$ is the indicator function of $\Gamma_{M}$, we can deduce a simple formula for the derivative of $J_{\alpha}$ :

$$
\frac{\mathrm{d}}{\mathrm{d} t} J_{\alpha}(\Sigma(t))=\int_{\Sigma}\left(-\nabla_{T} u \cdot \nabla_{T} w+\alpha \kappa\right) V_{n} \mathrm{~d} s .
$$

The Hadamard speed method consists in choosing the velocity $V_{n}$ such that a steepest descent (or a gradient flow) is achieved with respect to an appropriate norm for the velocity $V_{n}$ (cf [7, 49]). As discussed in [7], we have several possibilities to choose the velocity via the speed method, in particular we consider the steepest in $L^{2}(\Sigma(t))$ and in $H^{\frac{1}{2}}(\Sigma(t))$. For the $L^{2}$-case we obtain the velocity on $\Sigma(t)$ as

$$
V_{n}=\nabla u \cdot \nabla w-\alpha \kappa:=V_{n}^{0}-\alpha \kappa, \quad \text { on } \Sigma(t),
$$

which corresponds to the classical version of the speed method and the standard approach for level set methods in inverse obstacle problems. Since preliminary numerical experiments indicate that this standard approach fails for the problem we consider, we use the gradient flow in the norm of $H^{\frac{1}{2}}(\Sigma(t))$, which led to reasonable results in [7]. In the case of the $H^{\frac{1}{2}}$-norm, we have additional smoothing of the velocity $V_{n}$ via

$$
\begin{aligned}
& \Delta \psi_{V_{n}}=0 \quad \text { in } \Omega \backslash \Sigma \\
& \frac{\partial \psi_{V_{n}}}{\partial n}=0 \quad \text { on } \Gamma_{N} \\
& \frac{\partial \psi_{V_{n}}}{\partial n}=\nabla u \cdot \nabla w-\alpha \kappa \quad \text { on } \Sigma(t) \\
& \psi_{V_{n}}=0 \quad \text { on } \Gamma_{D} \text {, }
\end{aligned}
$$

and then obtain the velocity as the trace $V_{n}=\psi_{V_{n}}$ on $\Sigma(t)$. Since $\psi_{V_{n}}$ is an extension of $V_{n}$ to the whole domain $\Omega$, we can use it directly as an extension velocity for the level set method, so that the resulting evolution equation is given by

$$
\frac{\partial \phi}{\partial t}+\psi_{V_{n}}|\nabla \phi|=0
$$

Other possible extensions of the velocity to at least a neighbourhood of $\Sigma(t)$ can be realized, e.g., by solving a stationary Hamilton-Jacobi equation as in [2]. Alternatively, we could also perform a strategy as for the $H^{\frac{1}{2}}$-case and solve the problem (3.6) with Dirichlet data $V_{n}^{0}$. Extensions of the curvature to the whole domain can be obtained as (cf [40]) 
$\kappa=\operatorname{div}\left(\frac{\nabla \phi}{|\nabla \phi|}\right)$, which finally leads to the level set evolution (with $V^{0}$ being an appropriate extension of $V_{n}^{0}$ )

$$
\frac{\partial \phi}{\partial t}+V^{0}|\nabla \phi|-\alpha|\nabla \phi| \operatorname{div}\left(\frac{\nabla \phi}{|\nabla \phi|}\right)=0 .
$$

For $\alpha=0$, this evolution is a first-order Hamilton-Jacobi equation with a non-local term caused by the dependence of $V^{0}$ on the zero level set; for $\alpha>0$, the evolution is of second order.

We want to mention that in the case $\alpha=0$, the associated least-squares problem is illposed, but we may still consider the speed method as an evolutive regularization method. As for any regularizing evolution for ill-posed problems in the presence of noise, an appropriate stopping criterion is needed relating the time $T_{*}$ at which the evolution is terminated with the noise level $\delta$ in (2.2). By analogy to the method of asymptotic regularization (cf [50]) and to a regularizing level set evolution for a class of shape reconstruction problems (cf [6] where a rigorous analysis has been carried out), we propose to use the discrepancy principle, choosing $T_{*}$ via

$$
T_{*}\left(\delta, \theta^{\delta}\right):=\inf \left\{t \in \mathbb{R}_{+}\left|\left\|\left.u(t)\right|_{\Gamma_{M}}-\theta^{\delta}\right\|_{L^{2}\left(\Gamma_{M}\right)} \leqslant \tau \delta\right\},\right.
$$

for appropriate (fixed) parameter $\tau>1$. We shall compare the results obtained with this regularization approach, which we call the terminated speed method, to those obtained with penalization by perimeter in the next section.

\subsection{Solving the Hamilton-Jacobi equation (3.1)}

For solving the Hamilton-Jacobi equation (3.1) numerically, we merely use well-known schemes. There is a vast literature on numerical solvers for Hamilton-Jacobi equations (e.g., see $[26,38,41])$ based on the idea of essentially non-oscillatory schemes.

In all our numerical tests, we used a fifth order WENO scheme to discretize the spatial part of the Hamilton-Jacobi equation and an explicit Runge-Kutta scheme of third order to solve the time part of the Hamilton-Jacobi equation (see [26]). Due to the finite domain, we added homogeneous Neumann boundary conditions to the Hamilton-Jacobi equation. The influence of this boundary condition can be ignored, however, since the inclusions do not touch the boundary in the cases under consideration.

The explicit time integration of the Hamilton-Jacobi equation requires the fulfilment of the CFL condition to maintain a stable Hamilton-Jacobi solver. This means that the time step $\Delta t$ is restricted by

$$
\Delta t \leqslant c \frac{h}{\left\|\psi_{V_{n}}\right\|_{L_{\infty}(\Omega)}},
$$

with a constant $c<1$, where $h$ denotes the mesh size. In all our numerical tests we chose $c=0.8$, which produced reasonable results.

According to theory the calculated velocity $V_{n}$ should result in a descent of the objective $J_{\alpha}(\Sigma)$ (equation (2.1). By using the CFL condition for the determination of the time step in the Hamilton-Jacobi solver, we observed that this might also result in an increase of the objective. This may be caused by the fact that the CFL-time step is too large or due to a poor approximation of the solution of the PDE (1.4) or the adjoint PDE (3.4). Note that one may obtain a high approximation error for the PDE (1.4) and the adjoint PDE (3.4) because of a poor approximation of the normal to the zero level set, which occurs if the level set function is highly oscillating close to the zero level set. In this case a reinitialization of the level set function may help. 
Obviously, an increase in the objective $J_{\alpha}(\Sigma)$ during the iteration is not desirable in an optimization context and hence, additionally to the CFL restriction, we restricted the time step $\Delta t$ such that a decrease in the objective is guaranteed. This was realized by implementing a simple line-search (bisection), up to some minimal step size (at most ten bisections), which nonetheless sometimes caused an increase in the objective. Due to the possible poor approximation of the normal at the zero level set, we additionally reinitialized the level set function to the signed distance function when the step size reached its minimum. Imposing all these restrictions on the time step the average behaviour of the objective $J_{\alpha}(\Sigma)$ decreases so that the remaining increases in the objective can be accepted.

Finally, we want to remark on the termination criterion for the perimeter regularization. Usually one would use a standard termination criterion from optimization for the regularized problem, e.g., terminate when

$$
d_{H}\left(\Sigma_{i+1}, \Sigma_{i}\right) \leqslant \epsilon,
$$

where $\epsilon$ is a small positive number. Our calculation of the Hausdorff distance has accuracy $d_{H}=O(h)$, so it is impossible to choose $\epsilon$ very small $(\epsilon<h)$. On the other hand, the choice $\epsilon \geqslant h$ would cause too early a termination of the algorithm. Hence, we chose to terminate according to a proper chosen number of iterations such that by experience the Hausdorff distance $d_{H}$ did not change.

\subsection{Solving the elliptic PDE (1.4)}

One of the major issues in the numerical solution of inverse obstacle problems, crack identification, shape optimization and also moving boundary problems is the type of discretization used for the underlying elliptic or parabolic partial differential equations, in particular the question of whether the mesh should be changed during the optimization or evolution process. Mainly three different approaches to this problem can be found in the literature, namely the finite element method, fictitious domain method and the immersed interface method. For our numerical simulations, we used the latter, mainly because of the theoretically well-studied properties, the fixed grid for all time (optimization) steps and due to the available Fortran sources for the IIM solver, provided by Li. One further advantage of the IIM in our application is that the finite difference grid can also be used to discretize the Hamilton-Jacobi equation (3.1).

All standard finite element discretizations of the PDE (1.4) face one problem in the level-set approach, namely the need for two different meshes, a triangular one for the elliptic equation and a rectangular finite difference grid for the Hamilton-Jacobi equation. This is mainly because almost all well-analysed discretization schemes for the Hamilton-Jacobi equation are based on finite difference discretization. It is usually not desirable to map quantities from one grid to another, which may introduce a loss in accuracy and further numerical instabilities. However, we want to remark that numerical tests with a finite-element based fictitious domain method lead to similar results as for the IIM.

The immersed interface method was originally developed to treat elliptic interface problems with a finite difference discretization. It is proved to be of optimal accuracy; furthermore the interface and the finite difference grid are independent. For more details see $[23,25,30,31]$. The IIM seems to be quite well suited for interface identification problems because the finite difference grid for the PDE can be fixed once and for all (no remeshing). Using a similar trick as in the fictitious domain method it is also possible to treat inclusion or boundary identification problems with the IIM. This procedure is described in more detail in section 3.3.1. 
3.3.1. Immersed interface method for inclusions. Originally, the IIM was developed to treat interface problems with a finite difference discretization (see [23, 25, 30]). To also solve inclusion or boundary problems (1.4) with the IIM we have to transform the inclusion or boundary problem to an interface problem. We do this transformation along the suggestions of Li et al [24, 31] for the inclusion problem (1.4) only.

First, we extend the solution of the inclusion problem (1.4) to the inclusion $\Omega_{-}$such that

$$
\Delta u=0 \quad \text { in } \quad \Omega_{-} .
$$

For this reason we can either use the extension in $H^{1}(\Omega)$, i.e. $\left.[u]\right|_{\Sigma}:=u_{+}-\left.u_{-}\right|_{\Sigma}=0$, or require the jump of the normal derivative $\left.\left[\frac{\partial u}{\partial n}\right]\right|_{\Sigma}$ at the inclusion to be zero. In this way we get naturally the following equivalent formulations of the inclusion problem (1.4):

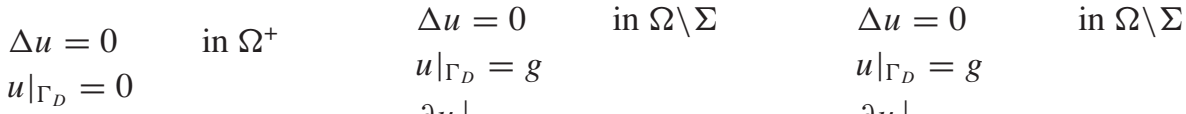

$$
\begin{aligned}
& \left.\frac{\partial u}{\partial n}\right|_{\Gamma_{N}}=\left.f \quad \Leftrightarrow \quad \frac{\partial u}{\partial n}\right|_{\Gamma_{N}}=\left.f \quad \Leftrightarrow \quad \frac{\partial u}{\partial n}\right|_{\Gamma_{N}}=f \\
& \begin{array}{lll}
\left.\frac{\partial u}{\partial n}\right|_{\Sigma}=0 & {\left.\left[\frac{\partial u}{\partial n}\right]\right|_{\Sigma}=-\frac{\partial u}{\partial n}} & {\left.\left[\frac{\partial u}{\partial n}\right]\right|_{\Sigma}=0 .}
\end{array}
\end{aligned}
$$

Solving any of these equivalent formulations (if meas $\left(\Gamma_{D}\right)>0$ ) is a well-posed problem (in particular unique). The last two formulations are interface problems in a fixed point form.

Introducing a new variable $w$ for either $-\frac{\partial u}{\partial n}-\left.\right|_{\Sigma}$ or $\left.u_{+}\right|_{\Sigma}$, we get a saddle point form that is equivalent to the original PDE (3.7)

$$
\begin{array}{lll}
\Delta u=0 \quad \text { in } \Omega \backslash \Sigma & \Delta u=0 \quad \text { in } \Omega \backslash \Sigma \\
\left.u\right|_{\Gamma_{D}}=g & \left.u\right|_{\Gamma_{D}}=g \\
\left.\frac{\partial u}{\partial n}\right|_{\Gamma_{N}}=f & \left.\frac{\partial u}{\partial n}\right|_{\Gamma_{N}}=f \\
{\left.[u]\right|_{\Sigma}=0} & \Leftrightarrow & {\left.[u]\right|_{\Sigma}-w=0} \\
{\left.\left[\frac{\partial u}{\partial n}\right]\right|_{\Sigma}-w=0} & {\left.\left[\frac{\partial u}{\partial n}\right]\right|_{\Sigma}=0} \\
\left.\frac{\partial u}{\partial n}\right|_{\Sigma}=0 & \left.u_{-}\right|_{\Sigma}=0 .
\end{array}
$$

Note that the restriction $\left.\frac{\partial u}{\partial n}\right|_{\Sigma}=0$ and $\left.u_{-}\right|_{\Sigma}=0$ has to be done in $H^{-\frac{1}{2}}(\Sigma)$ and $H^{\frac{1}{2}}(\Sigma)$.

Next we discretize this system according to the IIM, i.e. the interface problem becomes

$$
A u+B w=F,
$$

where $A$ is the discretization of the Laplacian (e.g., 5-point stencil) and $B, F$ are calculated due to the rules for IIM (see Li [31]). As in the work of Li et al [24, 31] we discretize the restriction $\left.\frac{\partial u}{\partial n_{+}}\right|_{\Sigma},\left.u_{+}\right|_{\Sigma}=0$ using some properties of the IIM resulting in

$$
C u+D w=G,
$$

where $C, D, G$ are matrices, respectively vectors, obtained from the immersed interface discretization. One requirement we do not meet, using this discretization of the restriction, is that we discretize the restriction in $L_{2}(\Sigma)$ (more accurate $l_{2}(\Sigma)$ ) instead of $H^{\frac{1}{2}}(\Sigma)$ or $H^{-\frac{1}{2}}(\Sigma)$. From tests done by $\mathrm{Li}[31]$ this does not seem to have much influence and so we nonetheless use this discretization. 
Finally, we can eliminate the variable $u$ by using the inverse of the operator $A$. Computationally, the application of this inversion can be realized very efficiently by fast Poisson solvers. When we substitute $u$ into the equation for the restriction we end up with a smaller Schur complement equation

$$
\left(D-C A^{-1} B\right) w=G-C A^{-1} F .
$$

We solve this equation by the generalized minimum residual method (GMRES). According to $\mathrm{Li}$ [31], the number of iterations to solve this equation with the GMRES is independent of the mesh size (which we also observed numerically for the inclusion problem) and so we obtain an efficient method to solve the inclusion problem (3.7). Clearly some analysis is missing, especially concerning the stability of the discrete system, i.e. whether or not the Schur complement can be inverted stable and independent of the mesh size.

\section{Remark 2.}

- The operators $A, A^{-1}, B, C, D$ are never calculated explicitly. Only their applications are needed, which makes computations efficient.

- For certain shapes, especially shapes with very high curvatures (locally), we observed that the solution to the discrete system does not fit the real solution very well. Probably this is either due to an instable calculation of the operators $B, C, D$ and vectors $F, G$ according to the IIM or due to a non-properly converging GMRES procedure caused by the wrong space for the restriction equation $\left(l_{2}(\Sigma)\right.$ instead of $\left.H^{\frac{1}{2}}(\Sigma), H^{-\frac{1}{2}}(\Sigma)\right)$.

- In our calculations we used the reformulated PDE (3.7) with the Dirichlet restriction $\left.u_{-}\right|_{\Sigma}=0$.

\section{Numerical results}

In the following, we shall report on some numerical experiments carried out with the methods described in the previous section to test the newly developed theory about the perimeter regularization as well as the proposed terminated speed method.

We restrict ourselves to the anti-planar case (1.4); because numerical tools are available for this case, similar results can be expected for the planar case. All performed tests are done with the same test configuration, i.e. we did all calculations on a square domain $\Omega=[-1,1] \times[-1,1]$ with fixed boundary conditions. Only the inclusion $\Omega_{-}$and the corresponding measurements vary from example to example. The geometric configuration, the boundary conditions and the problem specification are given in the following picture:

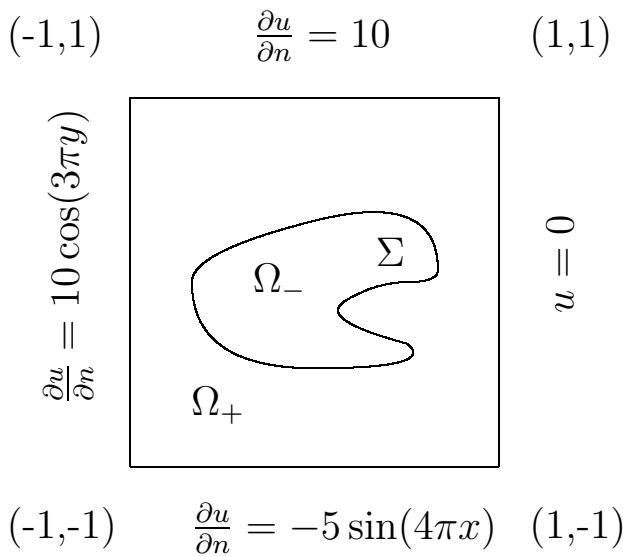

$$
\begin{aligned}
& \Delta u=0 \quad \text { in } \Omega_{+} \\
& \left.u\right|_{\Gamma_{D}}=0 \\
& \left.\frac{\partial u}{\partial n}\right|_{\Gamma_{N}}=h \\
& \left.\frac{\partial u}{\partial n}\right|_{\Sigma}=0
\end{aligned}
$$

Measurements :

$$
\theta^{\delta}=\left.u\right|_{\Gamma_{N}} \text { in } L_{2}\left(\Gamma_{N}\right)
$$


For the starting value $\phi_{0}$ of the Hamilton-Jacobi equation (3.1), we chose for all test examples $\phi_{0}=x^{2}+y^{2}-0.3^{2}$, i.e. a level-set function having as zero level line a circle with centre $(0$, $0)$ and radius 0.3 .

To avoid inverse crimes (see comments by Colton and Kress [12, p 133]), we calculated our measurements on a very fine grid (e.g., $2049 \times 2049$ ), having no grid points in common with the one used later for the numerical solutions of the inverse problem. These artificial measurements were projected by linear interpolation to the grid for the inverse problem.

The stability and convergence of the terminated speed method as well as the perimeter regularization were tested by perturbing the measurements with some artificial noise (of levels $\delta=5,3,1,0.8,0.5,0.3,0.1 \%)$. We generated this noise using Gaussian random values for every measurement point. Then we scaled this artificially produced noise by its $L_{2}\left(\Gamma_{N}\right)$-norm, which was added, scaled by its (normalized) noise level $\frac{\delta\|u\|_{\Gamma_{N}}}{100}$, to the (exact) measurements.

Remark 3. Even for exact measurements our solver faces noisy data. This is due to the (coarse) discretization of the PDE. For the discretization we chose, we expect approximately an additional noise of $\sim 0.1 \%$.

Terminated speed method: The discrepancy principle was chosen to terminate with $\tau=2$, i.e. solve the Hamilton-Jacobi equation (3.1) as long as $\left\|u(T)-\theta^{\delta}\right\|_{L_{2}\left(\Gamma_{M}\right)} \geqslant 2 \delta\left\|\theta^{\delta}\right\|_{L_{2}\left(\Gamma_{N}\right)}$.

Perimeter regularization: For the perimeter regularization we chose the penalization parameter $\alpha$ to be proportional to the noise level, i.e. $\alpha=c \delta$. According to the theory developed in section 2.1, this is a stable and converging method. Since we do not have a useful rule of how to chose the constant $c$, we used $c=5$ for all our test examples (corresponding merely to the scaling of the problem).

For almost all tests we plotted the Hausdorff distance $d_{H}\left(\Sigma_{\alpha}^{\delta}, \Sigma^{*}\right)$ versus the noise level $\delta$ (in logarithmic scale). Additional to the Hausdorff distance which is a very strong measure to compare the quality of several identification results, we also plotted the $L_{1}$ distance, i.e.

$$
d_{1}\left(\Sigma_{\alpha}^{\delta}, \Sigma^{*}\right)=\int_{\Omega}\left|\chi_{\left\{\hat{\phi}_{\alpha}^{\delta} \leqslant 0\right\}}-\chi_{\{\hat{\phi} \leqslant 0\}}\right| \mathrm{d} x,
$$

versus the noise level $\delta$ (logarithmic scale).

For inverse problems there are usually no benchmark examples available. Hence, we chose our test examples such that they are somehow representative for this class of problems, not too simple and challenging for the algorithm.

\subsection{Ellipse}

For the first test example, the inclusion is an ellipse with aspect ratio 5/3.

$$
\Sigma^{*}=\left\{(x, y) \mid\left(\frac{x}{0.5}\right)^{2}+\left(\frac{y-0.1}{0.3}\right)^{2}=1\right\},
$$

This is probably the simplest example so we can expect the algorithm to perform well. For the discretization of the PDE (3.7) and the Hamilton-Jacobi equation (3.1), we chose a $257 \times 257$ grid. In figure 2 , we present the calculated inclusions for the noise levels $\delta=5,1,0.5,0.1 \%$ as well as the exact shape for both the terminated speed method and the perimeter regularization. One observes that the reconstructed inclusion approaches the exact inclusion with decreasing noise level, as predicted by theory. Only parts of the inclusion close to the Dirichlet boundary and close to the Neumann boundary with constant load are of poor quality. For the part close to the Dirichlet boundary this is not unexpected, since here we 


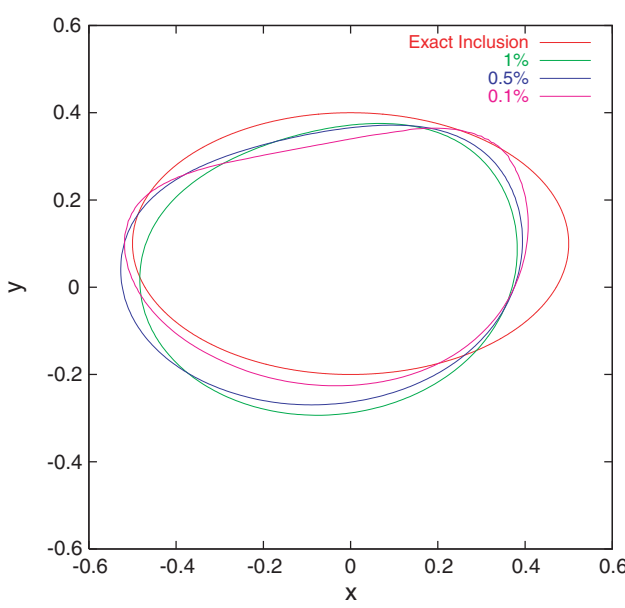

(a)

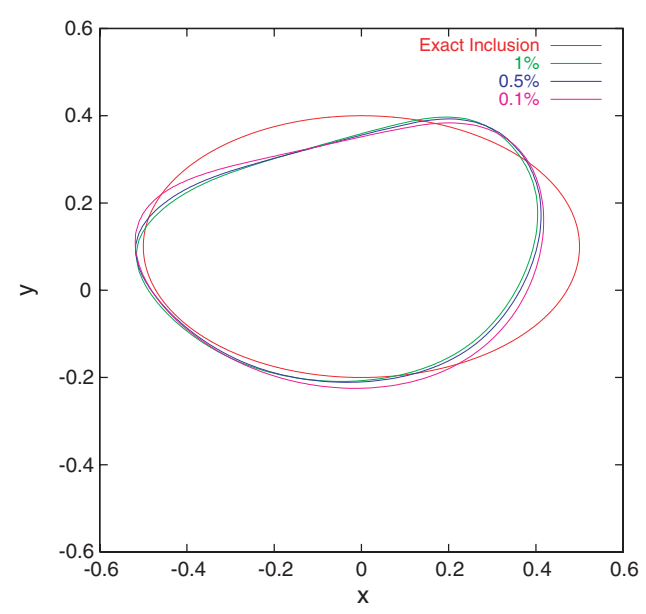

(b)

Figure 2. Ellipse: identified inclusion for $\delta=1,0.5,0.1 \%$. (a) Terminated speed method. (b) Perimeter regularization.

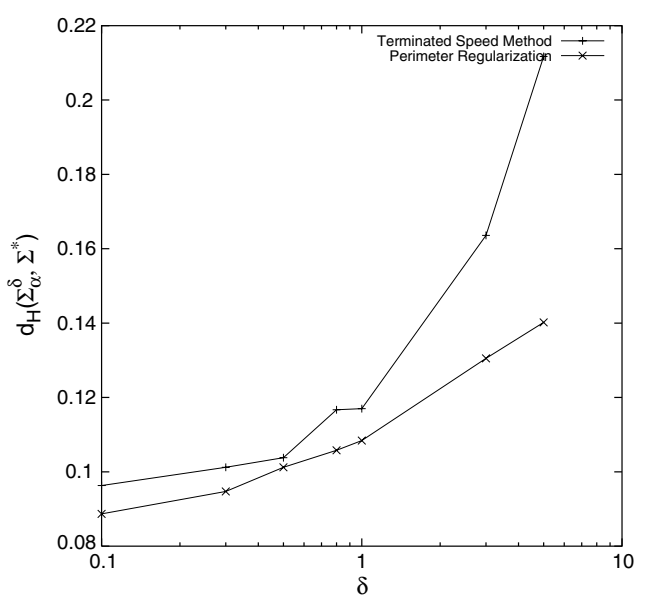

(a)

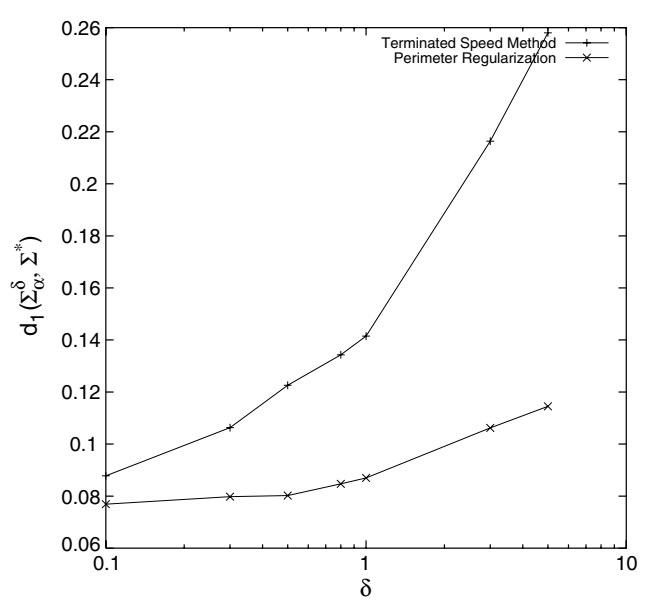

(b)

Figure 3. Ellipse: convergence in Hausdorff and $L_{1}$ distance versus noise $\delta$. (a) Hausdorff distance $d_{H}\left(\Sigma_{\alpha}^{\delta}, \Sigma^{*}\right)$. (b) $L_{1}$ distance $d_{1}\left(\Sigma_{\alpha}^{\delta}, \Sigma^{*}\right)$.

have no measurements and so the objective will not be very sensitive to changes close to this boundary. In figure 3 , we plotted the Hausdorff distance $d_{H}$, respectively the $L_{1}$-distance $d_{1}$, with respect to the noise level $\delta$. The figure confirms what we already recognized in figure 2, that the quality of the identified inclusion $\Sigma_{\alpha}^{\delta}$ improves with decreasing noise level. Both measures, the Hausdorff distance and the $L_{1}$-distance, decrease with the noise level $\delta$.

\subsection{Sharp ellipse}

In the second test example also, the inclusion is also an ellipse but with large aspect ratio 6 .

$$
\Sigma^{*}=\left\{(x, y) \mid\left(\frac{x}{0.6}\right)^{2}+\left(\frac{y-0.1}{0.1}\right)^{2}=1\right\} \text {. }
$$




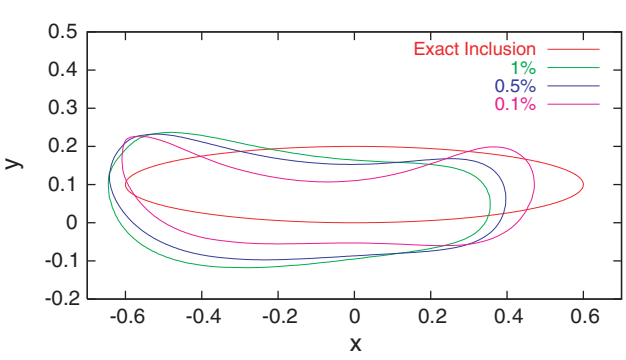

(a)

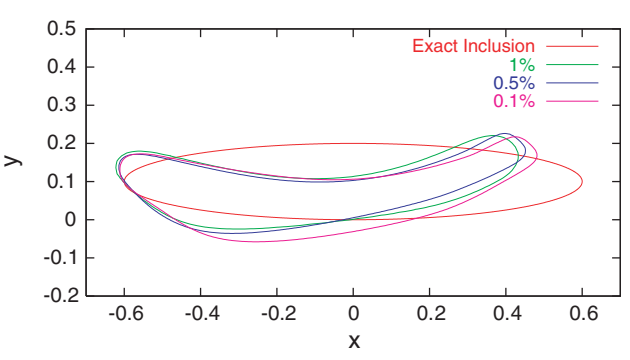

(b)

Figure 4. Sharp ellipse: identified inclusion for $\delta=5,1,0.1 \%$. (a) Terminated speed method. (b) Perimeter regularization.

The large aspect ratio causes a rather large curvature which is a challenge for the PDE solver. Also, the inverse solver suffers from this large curvature. This time we discretize the PDE (3.7) and the Hamilton-Jacobi equation (3.1) by a $513 \times 513$ grid in order to obtain a better resolution of the geometry. Only noise levels above $\delta \geqslant 1 \%$ were done on a $257 \times 257$ grid.

Note that due to the large aspect ratio of the ellipse for a $257 \times 257$ grid, there are only a few grid points in the inclusion, which is slightly dangerous for the immersed interface discretization. As before, we present in figure 4 the reconstructed inclusions for the noise levels $\delta=5,1,0.1 \%$ as well as the exact shape $\Sigma^{*}$ for both the terminated speed method and the perimeter regularization. Also here the reconstruction improves with decreasing noise. Nonetheless, the reconstruction is still of poor quality for the finest noise level. The length and the width of the ellipse are almost correct but the curvature at the sharp radius is completely far off for the terminated speed method whereas the identified shape by the perimeter regularization becomes too narrow. One may expect that for high curvature the reconstructed shape will not be very accurate, but surprisingly now the part of the interface with small curvature that is close to the Neumann boundary with constant load is far off the exact solution. For the same reason as for the ellipse with moderate aspect ratio, the reconstruction close to the Dirichlet boundary is of poor quality.

Despite the poor quality of the reconstruction in figure 4 , the error of the reconstructions in the Hausdorff distance decreases, as seen in figure 5, with the noise level. This is again in accordance with our theory about the perimeter regularization and confirms the terminated speed method. For the perimeter regularization, the $L_{1}$-distance seems to increase but this is not in contradiction to the theory developed before which is only valid for the Hausdorff distance $d_{H}$.

\subsection{Star shaped}

Up to now, all test examples were convex which is usually quite a nice property. In order to test a non-convex shape our third example is a star-shaped inclusion.

$\Sigma=\left\{(x, y) \mid x^{2}+(y-0.1)^{2}=\left(0.2+0.1 * \exp ^{1.4 * \cos (3 * \kappa-2)+0.4 * \sin \kappa}\right)^{2} \kappa=\arctan \frac{x}{y-0.1}\right\}$.

The star-shaped inclusion itself has again quite a large curvature and additionally the curvature changes sign. So we can expect that this test example is quite difficult for the algorithm. Especially from the last two examples we cannot expect reconstructions of high quality in the presence of noise. 


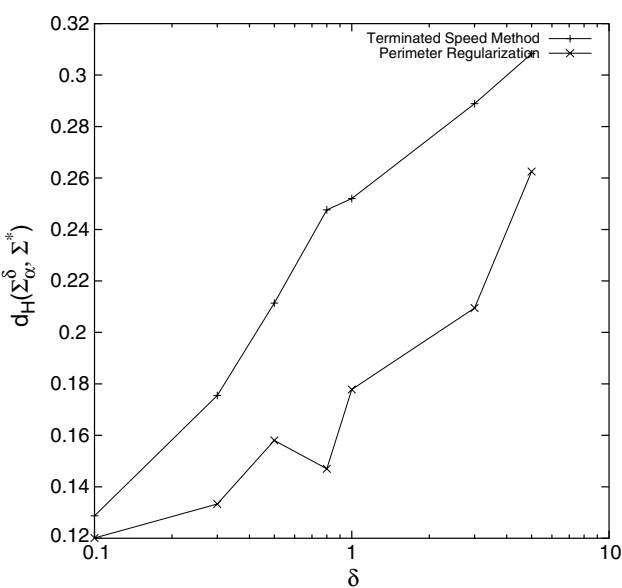

(a)

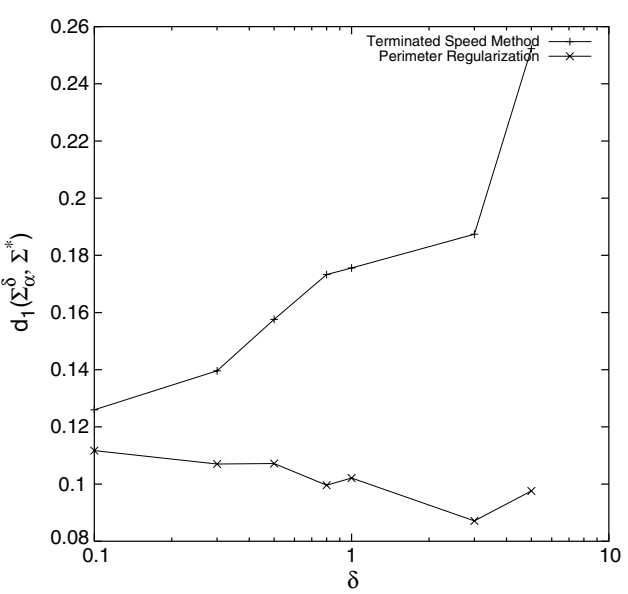

(b)

Figure 5. Sharp ellipse: convergence in Hausdorff and $L_{1}$ distance versus noise $\delta$. (a) Hausdorff distance $d_{H}\left(\Sigma_{\alpha}^{\delta}, \Sigma^{*}\right)$. (b) $L_{1}$ distance $d_{1}\left(\Sigma_{\alpha}^{\delta}, \Sigma^{*}\right)$.

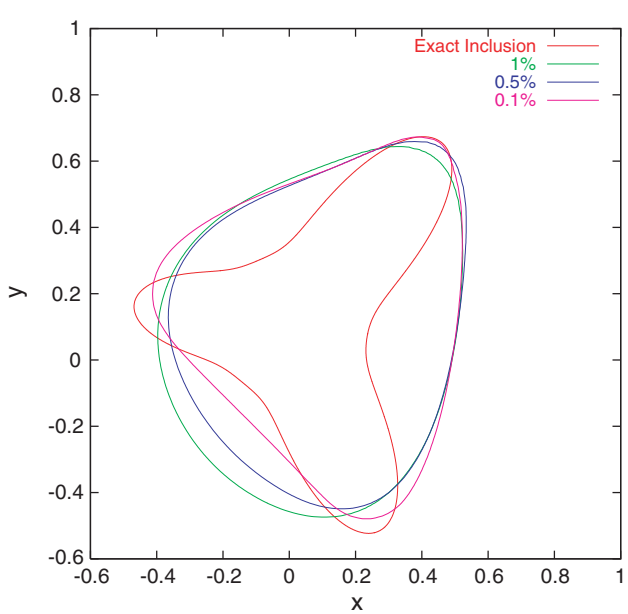

(a)

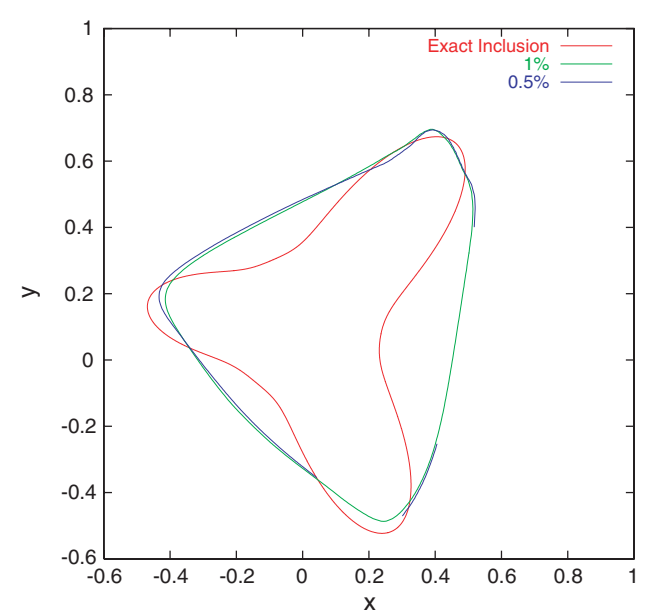

(b)

Figure 6. Star shape: identified inclusion for $\delta=5,1,0.1 \%$. (a) Terminated speed method. (b) Perimeter regularization.

For the discretization of the PDE (3.7) and the Hamilton-Jacobi equation (3.1), we chose a $257 \times 257$ grid.

As expected we observe in figure 6 that the reconstructions get very poor for both the perimeter regularization and the terminated speed method. Even worse, figure 7 indicates that the perimeter regularization is not converging which would contradict the theory. Fortunately, we are so far off the solution that we can argue that the result does not contradict the theory which is only a local result. Despite this negative result one must be aware of the fact that the inclusion detection problem from a single boundary measurement is probably severely ill-posed, so we could not expect much better reconstructions.

We finally remark that even for visually poor reconstructions (cf figure 6) and increasing Hausdorff and $L_{1}$ distance with decreasing noise level, the reconstruction is improved locally 


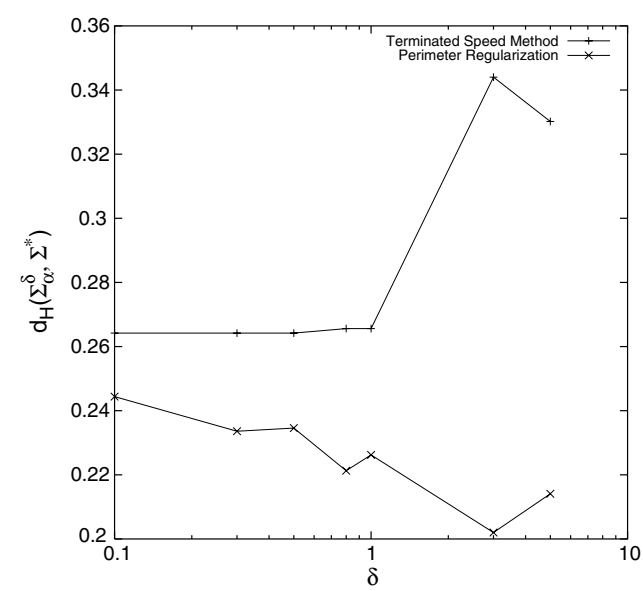

(a)

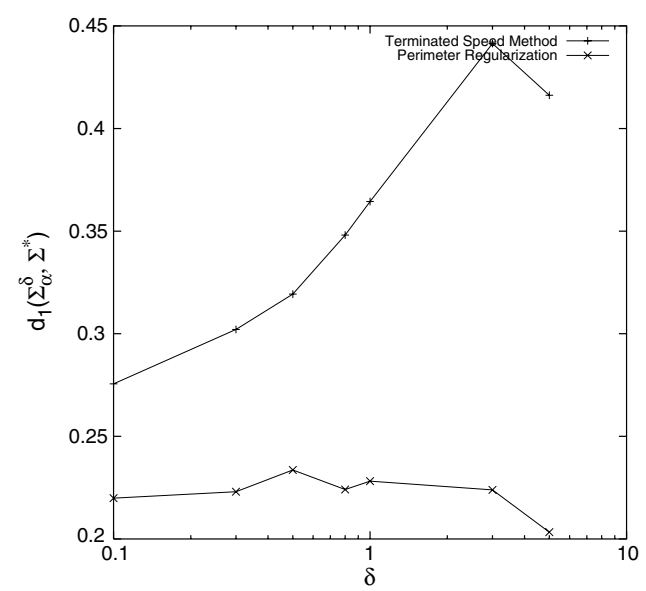

(b)

Figure 7. Star shaped: convergence in Hausdorff and $L_{1}$ distance versus noise $\delta$. (a) Hausdorff distance $d_{H}\left(\hat{\Sigma}_{\alpha}^{\delta}, \hat{\Sigma}^{*}\right)$. (b) $L_{1}$ distance $d_{1}\left(\hat{\Sigma}_{\alpha}^{\delta}, \hat{\Sigma}^{*}\right)$.

at the three arms of the star. The curvature of the reconstructed inclusion also starts to change sign, when the noise is low enough. Hence, one would expect much better reconstructions for significantly lower noise levels, which seems to be unrealistic in practice. The only way to improve the quality of the reconstruction seems to be the use of multiple measurements (i.e. measurements for different Neumann values). The application of level set methods in this case is an interesting and important topic for future research.

\subsection{Two circles}

Our final example is probably the most challenging one. The inclusion consists of two circles, which can in principle be handled by the level set method as opposed to most classical shape reconstruction algorithms based on parametrizations. Again the PDE (3.7) and the HamiltonJacobi equation (3.1) are approximated by a $257 \times 257$ grid.

$$
\Sigma=\left\{(x, y) \mid(x+0.4)^{2}+(y-0.4)^{2}=0.3^{2} \text { or }(x-0.3)^{2}+(y+0.3)^{2}=0.4^{2}\right\} .
$$

The identifiability result in [4] would theoretically allow us to identify these multiply connected inclusions uniquely, but from the numerical tests in the previous examples it seems doubtful if this is possible in the presence of noise. Indeed, the numerical results (cf figure 8) show that the initial domain does not split, but the algorithm shows at least a correct behaviour, i.e. the Hausdorff distance between exact and reconstructed shape decreases with $\delta$, and the shape seems to converge towards the correct inclusion.

For the terminated speed method, we were not able to terminate according to the termination criterion for low noise levels $\delta$, it seems that the evolution got stuck in a local minimum. This indicates that the reconstruction obtained with the perimeter regularization (looking similar) might also be a local minimum and not a global one. Figure 8 presents the calculated inclusions for noise levels $\delta=1,0.5,0.1 \%$ as well as the exact shape for the perimeter regularization. Visually, the reconstructions seem to get better when the noise $\delta$ reduces but still the components do not split into two components. As for the starshaped problem (section 4.3), figure 9 indicates that the Hausdorff distance for the perimeter regularization increases with the noise. This is either caused by getting stuck in a local minima 


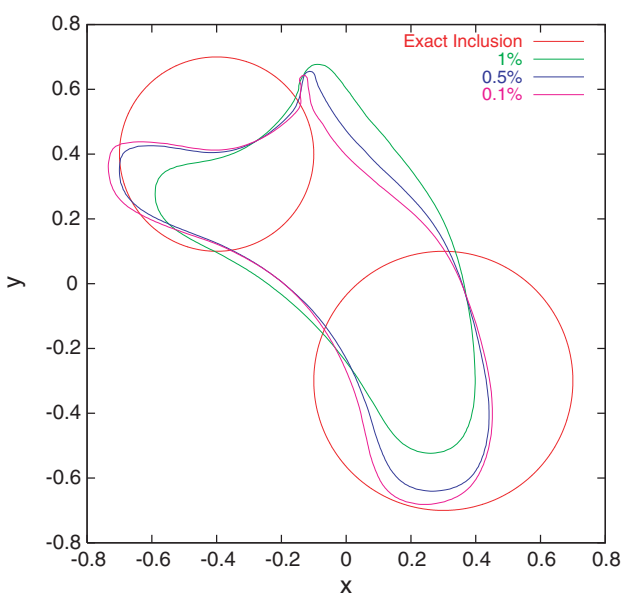

(a)

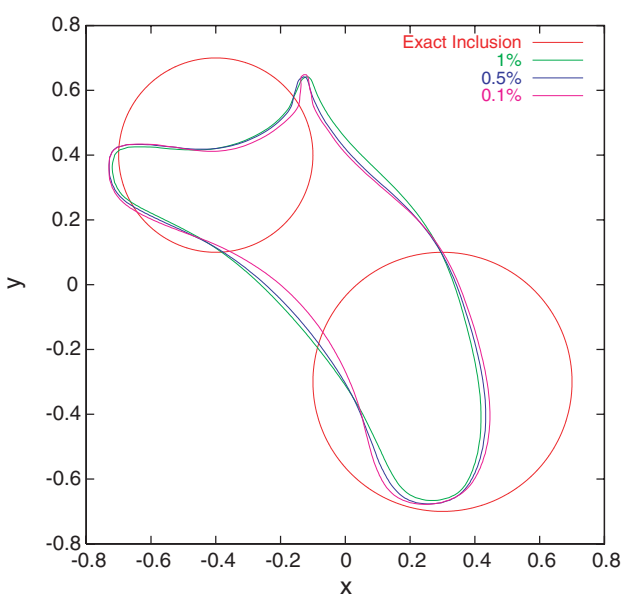

(b)

Figure 8. Two circles: identified inclusion for $\delta=1,0.5,0.1 \%$. (a) Terminated speed method. (b) Perimeter regularization.

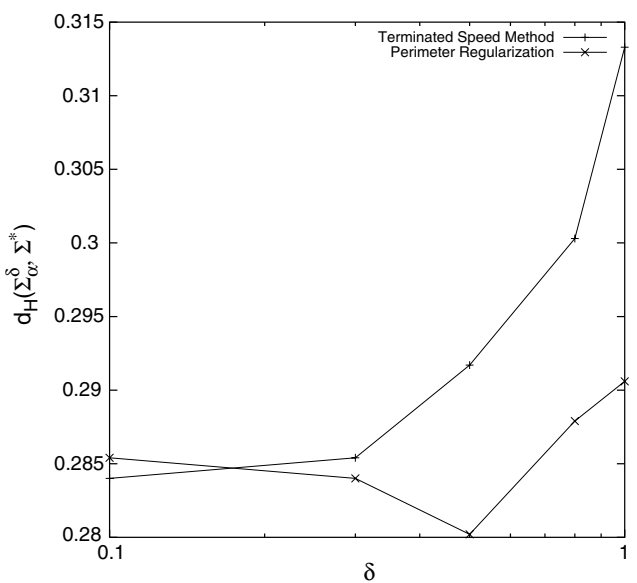

(a)

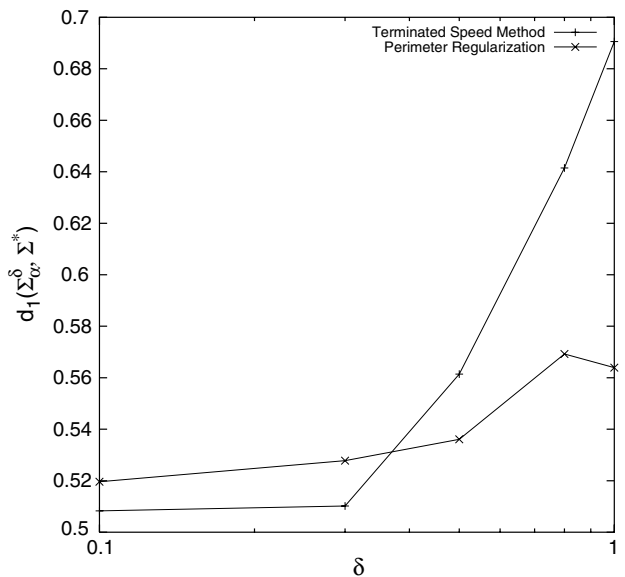

(b)

Figure 9. Two circles: convergence in Hausdorff and $L_{1}$ distance versus noise $\delta$. (a) Hausdorff distance $d_{H}\left(\Sigma_{\alpha}^{\delta}, \Sigma^{*}\right)$. (b) $L_{1}$ distance $d_{1}\left(\Sigma_{\alpha}^{\delta}, \Sigma^{*}\right)$.

or since the reconstructions are just too far from the exact solution to allow the application of the theoretical results.

\section{Conclusions and open problems}

We have presented a general approach to geometric inverse problems in linear elasticity, for which we also provided a convergent regularization method under very general geometric assumptions. Moreover, we have discussed the numerical solution of these inverse problems with some additional regularity assumptions for the geometry, using the level set method, which allows us to consider general shapes and does not rely on any parametrization. 
The numerical results obtained for the anti-planar case are encouraging for future research. Especially an extension to more than one set of boundary measurements seems to be interesting. From related problems such as impedance tomography and inverse scattering with full measurements (Dirichlet to Neumann map) it is well known that the twodimensional problem is not overdetermined, whereas the three-dimensional problem is. Hence, a possible application of the inclusion detection problem to the general three-dimensional case (with full measurements), which is also of importance in practice, may produce better results.

For the terminated speed method, an analysis is still missing but the numerical results compared to the yet theoretically analysed perimeter regularization give good evidence to proceed with analysing this method.

Moreover, many theoretical questions related to the solution by the level set method are still open, such as the well posedness of this evolution and its regularizing properties. In particular, for regular shapes there is some hope that we might apply local stability results obtained in [4] to obtain a convergence rate of the level set evolution, since the evolution uses exactly a path determined by the shape derivative, to which the stability estimates can be applied.

Analysis and computation are still open in the three-dimensional case, but we hope to obtain stability results in a similar way as for the two-dimensional planar case. Moreover, analogous questions are still open for thermo-elasticity, where the structural mechanics problems are coupled to a heat equation or for other types of material laws.

\section{Acknowledgments}

We thank Professor Heinz W Engl (University Linz) for stimulating this collaboration and for useful discussion. Moreover we thank Professor Zhilin Li (UNC Charlotte) for providing a code for the solution of a Dirichlet problem for the Laplace equation by the immersed interface method, which we could use with some modifications for the solution of the direct problem in the anti-planar case. Financial support is acknowledged to the Austrian National Science Foundation FWF under grant SFB F 013/08 and P13478-INF.

\section{References}

[1] Acar R and Vogel C 1994 Analysis of bounded variation penalty methods for ill-posed problems Inverse Problems 10 1217-29

[2] Adalsteinsson B and Sethian J A 1999 The fast construction of extension velocities in level set methods J. Comput. Phys. 148 2-22

[3] Allaire G, Jouve F and Toader A M 2002 A level-set method for shape optimization C. R. Acad. Sci., Paris 334 1125-30

[4] Ben Ameur H, Burger M and Hackl B 2003 On some geometric inverse problems in linear elasticity UCLA CAM-03-55

[5] Bryan K and Vogelius M 1992 A uniqueness result concerning the identification of a collection of cracks from finitely many electrostatic boundary measurements SIAM J. Math. Anal. 23 950-8

[6] Burger M 2001 A level set method for inverse problems Inverse Problems 17 1327-56

[7] Burger M 2003 A framework for the construction of level-set methods for shape optimization and reconstruction Interfaces Free Boundaries 5 301-29

[8] Cahn J W, Handwerker C A and Taylor J E 1992 Geometric models of crystal growth Acta Metall. Mater. 40 1443-72

[9] Chan T F, Chung E T and Tai X-C 2003 Electrical impedance tomography using level set representation and total variation regularization UCLA CAM-03-64 
[10] Chan T F and Tai X-C 2003 Level set and total variation regularization for elliptic inverse problems with discontinuous coefficients J. Comput. Phys. 193 40-66

[11] Chambolle A 2003 A density result in two-dimensional linearized elasticity and applications Arch. Ration. Mech. Anal. 167 211-33

[12] Colton D and Kress R 1998 Inverse Acoustic and Electromagnetic Scattering Theory 2nd edn, ed J E Marsden and L Sirovich (Berlin: Springer)

[13] Crandall M, Ishii H and Lions P L 1992 User's guide to viscosity solutions of second order partial differential equations Bull. Am. Math. Soc. 27 1-67

[14] Delfour M C and Zolésio J-P 2001 Shapes and geometries. Analysis, differential calculus, and optimization Advances in Design and Control vol 4 (Philadelphia, PA: SIAM) pp xvii, 482

[15] Dervieux A and Palmerio B 1975 Une formule de Hadamard dans les problèmes dídentification de domaine C.R. Acad. Sci., Paris A 280 1697-700

[16] Engl H W, Hanke M and Neubauer A 1996 Regularization of Inverse Problems (Dordrecht: Kluwer)

[17] Engl H W, Kunisch K and Neubauer A 1989 Convergence rates for Tikhonov regularisation of nonlinear ill-posed problems Inverse Problems 5 523-40

[18] Federer H 1969 Geometric Measure Theory (Berlin: Springer)

[19] Giacomini A 2002 Generalization of Golab's theorem and applications to fracture mechanics Math. Models Methods Appl. Sci. 12 1245-67

[20] Hettlich F and Rundell W 1996 Iterative methods for the reconstraction of an inverse potential problems Inverse Problems 12 251-66

[21] Hettlich F and Rundell W 1997 Recovery of the support of source term in elliptic differential equation Inverse Problems 13 959-76

[22] Hettlich F and Rundell W 1998 The determination of a discontinuity in a conductivity from a single boundary measurement Inverse Problems 14 67-82

[23] Huang H and Li Z 1999 Convergence analysis of the immersed interface method IMA J. Numer. Anal. 19 583-608

[24] Ito K, Kunisch K and Li Z 2001 Level-set function approach to an inverse interface problem Inverse Problems 17 1225-42

[25] Ito $\mathrm{K}$ and $\mathrm{Li} \mathrm{Z} 2001$ Maximum principle preserving schemes for interface problems with discontinuous coefficients SIAM J. Sci. Comput. 23 339-61

[26] Jiang G S and Peng D 2000 Weighted ENO schemes for Hamilton-Jacobi equations SIAM J. Sci. Comput. 21 2126-43

[27] Kirsch A 2000 The detection of holes by elasto-static measurements GAMM-Mitteilungen 23 79-92

[28] Kohn R V and Vogelius M 1985 Determining conductivity by boundary measurements; interior results Commun. Pure Appl. Math. 38 644-67

[29] Kunisch K and Pan X 1994 Estimation of interfaces from boundary measurements SIAM J. Control Opt. 32 $1643-74$

[30] LeVeque R and Li Z 1994 The immersed interface method for elliptic equations with discontinuous coefficients and singular sources SIAM J. Numer. Anal. 31 1019-44

[31] Li Z 1998 A fast iterative algorithm for elliptic interface problems SIAM J. Numer. Anal. 35 230-54

[32] Li Z 1998 The immersed interface method using a finite element formulation Appl. Numer. Math. 27 253-67

[33] Lions P L 1982 Generalized Solutions of Hamilton-Jacobi Equations (Boston, MA: Pitman)

[34] Litman A, Lesselier D and Santosa F 1998 Reconstruction of a two-dimensional binary obstacle by controlled evolution of a level-set Inverse Problems 14 685-706

[35] Dal Maso G and Toader R 2002 A model for the quasi-static growth of brittle fractures: existence and approximation results Arch. Ration. Mech. Anal. 162 101-35

[36] Morel J M and Solimini S 1994 Variational methods in image segmentation with 7 image processing experiments (Basel: Birkhäuser)

[37] Murat F and Simon J 1976 Sur le contrôle par un domaine géometrique (Publication du Laboratoire d'Analyse Numerique, Université de Paris VI)

[38] Osher S and Fedkiw R P 2002 Level Set Methods and Dynamic Implicit Surfaces (New York: Springer)

[39] Osher S and Santosa F 2001 Level set methods for optimization problems involving geometry and constraints I. Frequencies of a two-density inhomogeneous drum J. Comput. Phys. 171 272-88

[40] Osher S and Sethian J A 1988 Fronts propagating with curvature-dependent speed: algorithms based on Hamilton-Jacobi formulations J. Comput. Phys. 79 12-49

[41] Osher S and Shu C W 1991 Higher-order essentially nonoscillatory schemes for Hamilton-Jacobi equations SIAM J. Numer. Anal. 28 907-22

[42] Ramananjaona C, Lambert M and Lesselier D 2001 Shape inversion from TM and TE real data by controlled evolution of level sets Inverse Problems 17 1585-95 
[43] Ramananjaona C, Lambert M, Lesselier D and Zolesio J P 2001 Shape reconstruction of buried obstacles by controlled evolution of a level set: from a min-max formulation to numerical experimentation Inverse Problems 17 1087-112

[44] Rudin L I, Osher S and Fatemi E 1992 Nonlinear total variation based noise removal algorithms Physica D 60 259-68

[45] Santosa F 1996 A level-set approach for inverse problems involving obstacles ESAIM: Control, Optim. Calc. Var. 1 17-33

[46] Seidman T I and Vogel C 1989 Well-posedness and convergence of some regularization methods for nonlinear ill posed problems Inverse Problems 5 227-38

[47] Sethian J A 1999 Level Set Methods and Fast Marching Methods 2nd edn (Cambridge: Cambridge University Press)

[48] Sethian J A and Wiegmann A 2000 Structural boundary design via level set and immersed interface methods J. Comput. Phys. 163 489-528

[49] Sokolowski J and Zolesio J P 1992 Introduction to Shape Optimization (Berlin: Springer)

[50] Tautenhahn U 1994 On the asymptotical regularization of nonlinear ill-posed problems Inverse Problems 10 1405-18 